\title{
COMMUNICATION
}

\section{Kystes osseux solitaires mandibulaires à présentations radiologiques atypiques : À propos de deux cas cliniques.}

\section{Provost $\mathrm{M}^{1,2, *}$, Nguyen $\mathrm{T}^{2}$, Radoï $\mathrm{L}^{1,2}$}

1 - Faculté de chirurgie dentaire Paris Descartes, département de médecine buccale et de chirurgie buccale, Montrouge, France

\section{Introduction}

Les kystes osseux solitaires sont des lésions intra-osseuses vides ou à contenu séro-hémorragique, retrouvées majoritairement au niveau des métaphyses des os longs des enfants et des adolescents (1). La variété des noms de cette lésion (kyste simple, traumatique, idiopathique, essentiel) reflète l'incertitude quant à son étiologie. Les localisations maxillo-faciales sont exceptionnelles et concernent préférentiellement la partie antéro-moyenne du corps de la mandibule (1). L'image radiologique typique est celle d'une lésion radio-claire homogène, uniloculaire, aux bords nets et irréguliers, qui amincit les corticales osseuses, sans les interrompre ; la lésion peut être à distance des racines des dents évoluées ou les envelopper (2). Cependant, des présentations radiologiques atypiques sont décrites et nécessitent le diagnostic différentiel avec d'autres kystes ou tumeurs des maxillaires (1).

\section{Observation}

Deux cas atypiques de kystes solitaires mandibulaires, tant par leur localisation goniaque et ramique, que par leur rapport avec une dent de sagesse incluse et leur aspect multiloculaire et cloisonné, sont rapportés.

Dans le premier cas, un patient de 17 ans était adressé par son chirurgien-dentiste traitant pour l'avulsion des 4 dents de sagesse incluses. L'orthopantomogramme (OPT) montrait une image radio-claire multiloculaire, bien délimitée, située au niveau du ramus mandibulaire droit, s'étendant de la 48 incluse à l'échancrure sigmoïde.

Dans le deuxième cas, une patiente de 16 ans était adressée par son orthodontiste pour l'avulsion des 4 premières molaires cariées. L'OPT montrait une lésion radio-claire mandibulaire droite, située au niveau du corps, de l'angle et du ramus, d'aspect multiloculaire et cloisonné, au contact des racines des 46 et 47 évoluées et de la 48 incluse.

Les principales hypothèses diagnostiques évoquées dans ces deux cas étaient : kyste folliculaire (rapport des 48 incluses avec les lésions), kératokyste odontogénique et améloblastome (localisation goniaque et ramique, aspect multiloculaire et cloisonné). Dans les deux cas, le diagnostic de kyste radiculo-dentaire a été exclu devant la vitalité des dents du secteur 4. La TDM a confirmé l'aspect mixte des lésions (images radio-claires avec présence de cloisons radio-opaques), ainsi que l'amincissement des corticales, sans rupture de celles-ci. L'intervention chirurgicale a consisté en la réalisation d'une 
ostéotomie corticale (permettant d'objectiver une cavité osseuse sans revêtement épithélial, à contenu hémorragique), un curetage appuyé des parois, et l'avulsion des dents incluses. Le suivi radiologique a montré une cicatrisation osseuse quasi-complète à 12 mois.

Discussion

Parmi la multitude de théories sur l'étiologie des kystes solitaires (dégénérescence tumorale, traumatisme et anomalie de croissance osseuse), la cause traumatique est la plus évoquée (1, 2). Dans les deux cas cliniques, l'antécédent de traumatisme était absent. Cependant, la présence d'une cavité osseuse au contenu hématique plaidait en faveur d'une hémorragie intra-osseuse, traumatique ou non.

Comme dans ces deux cas, le kyste solitaire est généralement asymptomatique et découvert fortuitement à un examen radiologique de routine (3). Les lésions ramiques, d'aspect multiloculaire et cloisonné, sont rares; elles peuvent être interprétées à tort comme améloblastomes ou kératokystes $(1,3)$.

\section{Conclusion}

La méconnaissance des présentations radiologiques atypiques des kystes solitaires peut conduire à des erreurs de diagnostic et à des attitudes thérapeutiques agressives et inappropriées.

\section{Références}

1 - Mervyn Shear, Paul Speight. Solitary bone cyst. In: Cysts of the Oral and Maxillofacial Regions, Fourthedition. Eds Blakwell Munskgaard, 2007; 156-61.

2 - Kaugars GE, Cale AE. Traumatic bone cyst. Oral Surg Oral Med Oral Pathol 1987;63:318-24.

3 - Copete MA, Kawamata A, Langlais RP. Solitary bone cyst of the jaws: radiographic review of 44 cases. Oral Surg Oral Med Oral Pathol Oral Radiol Endod 1998;85:221-5. 